\title{
Variational Multiscale Element Free Galerkin Method Coupled with Low-Pass Filter for Burgers' Equation with Small Diffusion
}

\author{
Ping Zhang, Xiaohua Zhang, and Laizhong Song \\ College of Science, China Three Gorges University, Yichang 443002, China \\ Correspondence should be addressed to Xiaohua Zhang; zhangxiaohua07@163.com
}

Received 22 October 2015; Revised 27 January 2016; Accepted 2 February 2016

Academic Editor: Stephen C. Anco

Copyright (C) 2016 Ping Zhang et al. This is an open access article distributed under the Creative Commons Attribution License, which permits unrestricted use, distribution, and reproduction in any medium, provided the original work is properly cited.

\begin{abstract}
Variational multiscale element free Galerkin (VMEFG) method is applied to Burgers' equation. It can be found that, for the very small diffusivity coefficients, VMEFG method still suffers from instability in the presence of boundary or interior layers. In order to overcome this problem, the high order low-pass filter is used to smooth the solution. Three test examples with very small diffusion are presented and the solutions obtained are compared with exact solutions and some other numerical methods. The numerical results are found in which the VMEFG coupled with low-pass filter works very well for Burgers' equation with very small diffusivity coefficients.
\end{abstract}

\section{Introduction}

Burgers' equation, which is a nonlinear partial differential equation, can model several physical phenomena such as traffic, shock waves, gas dynamics, longitudinal elastic waves in an isotropic solid, and turbulence problems. In general, the exact solution of Burgers' equation can be only obtained in special case. Moreover, in many cases these solutions involve infinite series which may converge very slowly for small diffusivity coefficients. Therefore, finding accurate and efficient numerical methods for solving Burgers' equation has been an attractive research undertaking. The main challenge of numerical solution of Burgers' equation lies in nonphysical oscillations for convection-dominated problem. Thus, many numerical schemes are constructed or developed in the last few years to solve Burgers' equation, such as finite difference method (FDM) [1-3], finite element method (FEM) [4-8], Bspline methods [9-11], and lattice Boltzmann method (LBM) [12]. A comprehensive review of the different methods for solving Burgers' equation can refer to [10].

In recent years, meshless methods emerge as a new numerical method for solving various mathematical-physical problems. Compared with the traditional computational methods based on mesh, meshless methods have many outstanding advantages. In these methods, the approximation is built only based on nodes and no predefined nodal connectivity is required; meanwhile, the removal and the addition of nodes in the domain are easily performed.

Previous studies $[13,14]$ show that the numerical solution may be corrupted by nonphysical oscillations when meshless methods are directly used to solving Burgers' equation with small diffusion. Usually, spatial stabilization technique is required for the meshless methods and many meshless stabilized methods are developed in recent years. Most of these stabilized methods are a generalization of the stabilized FEM [13-17], such as streamline-upwind Petrov-Galerkin (SUPG), Galerkin least-square (GLS), and subgrid scales (SGS). As Fries and Matthies [16] had pointed out, standard stabilization approaches in FEM can be directly applied to the meshless methods; however, the choice of the stabilization parameter required special attention. But in practice, the choice of the stabilization parameter is more or less dependent on the researches' experience. In order to avoid the selection of the stabilization parameter, Zhang and his coworkers [18] presented variational multiscale element free Galerkin method (VMEFG) which is free of user-defined stability parameters and a consistent stabilized method. Subsequently, they applied the method to solve Stokes problems [18], water wave problems [19], MHD flows problems [20], and convection-diffusion-reaction problem [21] successfully. But, as far as the solution of Burgers' equation is concerned, for the very small diffusivity coefficients, the VMEFG method 
also produces the numerical pseudo oscillations as we will see in the paper; therefore, we must find another technique to deal with it.

In recent years, high order low-pass filters have been proposed to treat hyperbolic conservation laws [22, 23] and some experiences show that a low-pass filter is ideal for suppressing the amplitude of undesirable high frequency components and does not affect the remaining components of the solution [24]. To the best of our knowledge, most numerical applications are restricted to problem involving moderate and larger diffusion. Moreover, no published results with meshless methods in conjunction with low-pass filter are used to solve Burgers' equations with very small diffusivity coefficients. Therefore, the main goal of the paper is to evaluate VMEFG coupled with high order low-pass filter for Burgers' equation with very small diffusion.

This paper is organized as follows. In Section 2, we list some elementary knowledge about the VMEFG method and low-pass filter. In Section 3, numerical experiments are performed using our proposed method. And the conclusions are presented in the Section 4.

\section{Numerical Algorithms}

Let $\Omega \subset \mathrm{R}^{\text {nd }}$ be an open bounded region with piecewise smooth boundary $\Gamma$. The number of space dimensions, nd, is equal to 1 or 2 . Burgers' equation is written as follows:

$$
\frac{\partial \mathbf{u}}{\partial t}+\mathbf{u} \cdot \nabla \mathbf{u}-\frac{1}{\operatorname{Re}} \nabla \cdot(\nabla \mathbf{u})=0 \quad \text { in } \Omega \times[0, T],
$$

where $\mathbf{u}$ is the dependent variable resembling the flow velocity and Re is the Reynolds number characterizing the size of viscosity. From (1), it can be obviously seen that Burgers' equation is a simplified form of the Navier-Stokes equation having viscosity and nonlinear convection term. When the diffusivity coefficient is very small, Burgers' equation models convection-dominated problem.

Let $\mathbf{w}$ represent the weight function for velocity $\mathbf{u}$; then the standard weak form of (1) is given as follows:

$$
(\mathbf{w}, \dot{\mathbf{u}})+(\mathbf{w}, \mathbf{u} \cdot \nabla \mathbf{u})+\left(\nabla \mathbf{w}, \frac{1}{\operatorname{Re}} \nabla \mathbf{u}\right)=0,
$$

where $\dot{\mathbf{u}}=\partial \mathbf{u} / \partial t,(\cdot, \cdot)=\int_{\Omega}(\cdot) d \Omega$, that is, the $L_{2}$ product of the indicated arguments over domain $\Omega$.

In the paper, the moving least-square (MLS) method is used to construct the shape functions of meshless method and the detailed MLS approximation can refer to [25]. In general, owing to that the MLS approximation does not possess the Kronecker Delta condition property, this makes the imposition of essential boundary conditions more complicated and time-consuming than that of FEM. Here we used a simple technique proposed by Zhang et al. [19] to enforce the essential boundary conditions; that is, the nodal influence domain of meshless method is extended to have arbitrary polygon shape. Their numerical results indicated that the shape functions almost possess the Kronecker delta function property when the dimensionless size of the nodal influence domain $\alpha$ approaches to 1 .
2.1. Variational Multiscale Element Free Galerkin Method. The VMEFG method is inheritance of the variational multiscale FEM which was offered by Hughes et al. [26] and the main idea of the method is to divide the unknown physical quantity into fine scale and coarse scale. Then it can use bubble functions to determine fine scale solution analytically and gets stabilization parameter naturally. Last, it substitutes the fine scale solution into coarse scale problem and then obtains the coarse scale solution numerically. In the following, the brief introduction of VMEFG is presented.

Step 1 (multiscale decomposition). Assume that the velocity $\mathbf{u}$ and its weight function $\mathbf{w}$ can be decomposed into coarse scale and fine scale, respectively; namely,

$$
\begin{aligned}
\mathbf{u} & =\overline{\mathbf{u}}+\widehat{\mathbf{u}}, \\
\mathbf{w} & =\overline{\mathbf{w}}+\widehat{\mathbf{w}},
\end{aligned}
$$

where $\overline{\mathbf{u}}$ and $\widehat{\mathbf{u}}$ are the coarse scale and fine scale for velocity $\mathbf{u}$ and $\overline{\mathbf{w}}$ and $\widehat{\mathbf{w}}$ are the coarse scale and fine scale for weight function $\mathbf{w}$, respectively.

Similar to literatures $[19,27]$, we further assume that $\widehat{\mathbf{u}}$ and $\widehat{\mathbf{w}}$, although nonzero within background integral cell $\Omega^{K}$, vanish identically over the $\Omega^{K}$ boundary when the factor $\alpha$ approaches to 1 :

$$
\begin{array}{ll}
\widehat{\mathbf{u}}=0 & \text { on } \partial \Omega^{K}, \\
\widehat{\mathbf{w}}=0 & \text { on } \partial \Omega^{K} .
\end{array}
$$

We now substitute the trial solutions equation (3) and the weighting functions equation (4) in the standard variational equation (2), and this becomes the point of departure from the conventional Galerkin formulations

$$
\begin{gathered}
(\overline{\mathbf{w}}+\widehat{\mathbf{w}}, \dot{\overline{\mathbf{u}}})+(\overline{\mathbf{w}}+\widehat{\mathbf{w}},(\overline{\mathbf{u}}+\widehat{\mathbf{u}}) \cdot \nabla(\overline{\mathbf{u}}+\widehat{\mathbf{u}})) \\
+\left(\nabla(\overline{\mathbf{w}}+\widehat{\mathbf{w}}), \frac{1}{\operatorname{Re}} \nabla(\overline{\mathbf{u}}+\widehat{\mathbf{u}})\right)=0 .
\end{gathered}
$$

Next, employing the linearity between coarse scale $\overline{\mathbf{w}}$ and fine scale $\widehat{\mathbf{w}},(7)$ can be split into coarse scale problem $\bar{W}$ and fine scale problem $\widehat{W}$ as follows.

The Coarse Scale Problem $\bar{W}$. Consider

$$
\begin{aligned}
& (\overline{\mathbf{w}}, \dot{\overline{\mathbf{u}}})+(\overline{\mathbf{w}},(\overline{\mathbf{u}}+\widehat{\mathbf{u}}) \cdot \nabla(\overline{\mathbf{u}}+\widehat{\mathbf{u}}))+\left(\nabla \overline{\mathbf{w}}, \frac{1}{\operatorname{Re}} \nabla(\overline{\mathbf{u}}+\widehat{\mathbf{u}})\right) \\
& \quad=0 .
\end{aligned}
$$

The Fine Scale Problem $\widehat{W}$. Consider

$$
\begin{aligned}
& (\widehat{\mathbf{w}}, \dot{\overline{\mathbf{u}}})+(\widehat{\mathbf{w}},(\overline{\mathbf{u}}+\widehat{\mathbf{u}}) \cdot \nabla(\overline{\mathbf{u}}+\widehat{\mathbf{u}}))+\left(\nabla \widehat{\mathbf{w}}, \frac{1}{\operatorname{Re}} \nabla(\overline{\mathbf{u}}+\widehat{\mathbf{u}})\right) \\
& \quad=0 .
\end{aligned}
$$

Obviously, the coarse and fine scale equations are in fact nonlinear equations because of the convection term. In general, to solve nonlinear equations we need to linearize them. Here, 
we substitute the nonlinear convective coefficient $\overline{\mathbf{u}}+\widehat{\mathbf{u}}$ in (8) and (9) by the last converged solution $\mathbf{u}^{c}$ from the fixed point iteration.

The Linearized Coarse Scale Problem $\bar{W}$. Consider

$$
\begin{aligned}
& (\overline{\mathbf{w}}, \dot{\overline{\mathbf{u}}})+\left(\overline{\mathbf{w}}, \mathbf{u}^{c} \cdot \nabla(\overline{\mathbf{u}}+\widehat{\mathbf{u}})\right)+\left(\nabla \overline{\mathbf{w}}, \frac{1}{\operatorname{Re}} \nabla(\overline{\mathbf{u}}+\widehat{\mathbf{u}})\right) \\
& \quad=0 .
\end{aligned}
$$

The Linearized Fine Scale Problem $\widehat{W}$. Consider

$$
\begin{aligned}
& (\widehat{\mathbf{w}}, \dot{\overline{\mathbf{u}}})+\left(\widehat{\mathbf{w}}, \mathbf{u}^{c} \cdot \nabla(\overline{\mathbf{u}}+\widehat{\mathbf{u}})\right)+\left(\nabla \widehat{\mathbf{w}}, \frac{1}{\operatorname{Re}} \nabla(\overline{\mathbf{u}}+\widehat{\mathbf{u}})\right) \\
& \quad=0 .
\end{aligned}
$$

Step 2 (solution of the fine scale problem). According to the literatures $[19,27]$, we next deduce the fine scale solution $\widehat{\mathbf{u}}$ by using the bubble functions. Let us now consider the fine scale part of the weak form $\widehat{W}$. Exploiting linearity of the solution slot in the first term and employing integration by parts to the second term on the left-hand side in (11), we get

$$
\begin{aligned}
\left(\widehat{\mathbf{w}}, \mathbf{u}^{c} \cdot \nabla \widehat{\mathbf{u}}\right)+\left(\nabla \widehat{\mathbf{w}}, \frac{1}{\operatorname{Re}} \nabla \widehat{\mathbf{u}}\right) \\
\quad=\left(\widehat{\mathbf{w}},-\dot{\overline{\mathbf{u}}}-\mathbf{u}^{c} \cdot \nabla \overline{\mathbf{u}}+\nabla \cdot\left(\frac{1}{\operatorname{Re}} \nabla \overline{\mathbf{u}}\right)\right)=(\widehat{\mathbf{w}}, \overline{\mathbf{r}}),
\end{aligned}
$$

where $\overline{\mathbf{r}}=-\dot{\overline{\mathbf{u}}}-\mathbf{u}^{c} \cdot \nabla \overline{\mathbf{u}}+\nabla \cdot((1 / \operatorname{Re}) \nabla \overline{\mathbf{u}})$. In this process, we have applied the integration by parts as well as the relevant assumptions such as (6). In addition, it is important to note that $\overline{\mathbf{r}}$ is the residual of Burgers' equation for the coarse scale. This is a crucial ingredient of the VMEFG method and ensures that the resultant formulation yields a consistent stabilized method.

In order to obtain fine scale solution $\widehat{\mathbf{u}}$ analytically from (12) and deduce the structure of the stability parameter $\boldsymbol{\tau}$, we use bubble functions similar to FEM context [27]. Without loss of generality, we assume that the fine scale $\widehat{\mathbf{u}}$ and $\widehat{\mathbf{w}}$ are represented via bubble functions over the $\Omega^{K}$ of the EFG shape function when factor $\alpha$ approaches to 1 :

$$
\begin{array}{ll}
\left.\widehat{\mathbf{u}}\right|_{K}=\left.b_{1}^{K}(\boldsymbol{\xi}) \boldsymbol{\beta} \longrightarrow \widehat{\mathbf{u}}_{i}\right|_{K}=b_{1}^{K}(\boldsymbol{\xi}) \boldsymbol{\beta}_{i}, & \text { on } \Omega^{K}, \\
\left.\widehat{\mathbf{w}}\right|_{K}=\left.b_{2}^{K}(\boldsymbol{\xi}) \boldsymbol{\longrightarrow} \longrightarrow \widehat{\mathbf{w}}_{i}\right|_{K}=b_{2}^{K}(\boldsymbol{\xi}) \boldsymbol{\gamma}_{i}, & \text { on } \Omega^{K},
\end{array}
$$

where $b_{1}^{K}$ and $b_{2}^{K}$ represent the bubble functions for the trial solution $\widehat{\mathbf{u}}$ and weight function $\widehat{\mathbf{w}}$ over the $\Omega^{K}$, respectively, and $\beta$ and $\gamma$ are their coefficients, respectively, $i=1, \ldots$, nd (nd indicates the dimension of the problem).

In the paper, for the $1 \mathrm{D}$ problem, the bubble functions fine scale trial solution and weighting function are given as follows [27]:

$$
\begin{aligned}
& b_{1}^{K}=\left(1-x^{2}\right), \quad-1 \leq x \leq 1, \\
& b_{2}^{K}(x)= \begin{cases}\left(\frac{1}{1+X_{b}}\right)(x+1), & -1 \leq x \leq X_{b}, \\
\left(\frac{-1}{1-X_{b}}\right)\left(x-X_{b}\right)+1, & -1 \leq x \leq X_{b},\end{cases}
\end{aligned}
$$

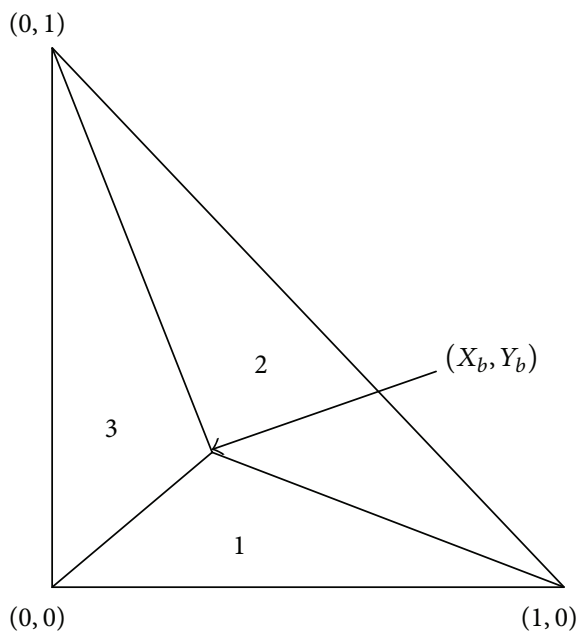

FIGURE 1: Schematic diagram showing the domains for the bubble functions for a reference triangle cell [21].

where $X_{b}$ is the location of the internal virtual node for the piecewise linear bubble and in the paper $X_{b}=-0.5$.

For the 2D problem, when the triangle background cells are used and in a reference background cell, the bubble for the fine scale trial solution is a quadratic bubble defined as [21]

$$
b_{1}^{K}(x, y)=27(1-x-y)
$$

For the fine scale weighting function, the reference background cell is divided into three regions (see Figure 1) and the bubble functions on these regions are given as follows:

$$
b_{2}^{K}(x, y)= \begin{cases}\frac{x y}{X_{b} Y_{b}} & \text { for } x, y \text { in region } 1, \\ \frac{1-x-y}{1-X_{b}-Y_{b}} & \text { for } x, y \text { in region 2, } \\ \frac{x y}{X_{b} Y_{b}} & \text { for } x, y \text { in region 3, }\end{cases}
$$

where $X_{b}$ and $Y_{b}$ represent the location of the internal virtual node in the background cell and in the paper $\left(X_{b}, Y_{b}\right)=$ $(0.34,0.34)$.

Substituting (13) and (14) into (12), and taking the constant coefficient vector $\beta$ and $\boldsymbol{\gamma}$ out of the integral expression, we have

$$
\begin{gathered}
\boldsymbol{\gamma}^{T}\left[\int_{K} b_{2}^{K} \mathbf{u}^{c} \cdot \nabla b_{1}^{K} d \Omega \mathbf{I}+\left(\int_{K} \frac{1}{\operatorname{Re}} \nabla b_{1}^{K}: \nabla b_{2}^{K} d \Omega \mathbf{I}\right.\right. \\
\left.\left.+\int_{K} \frac{1}{\operatorname{Re}} \nabla b_{1}^{K} \otimes \nabla b_{2}^{K} d \Omega\right)\right] \boldsymbol{\beta}=\gamma^{T}\left(b_{2}^{K}, \overline{\mathbf{r}}\right) .
\end{gathered}
$$

Since $\gamma$ is arbitrary, consequently we get

$$
\beta=\mathrm{S}^{-1} \mathbf{R}
$$


where $\mathbf{S}$ and $\mathbf{R}$ are defined as follows:

$$
\begin{aligned}
\mathbf{S} & =\int_{K} b_{2}^{K} \mathbf{u}^{c} \cdot \nabla b_{1}^{K} d \Omega \mathbf{I}+\left(\int_{K} \frac{1}{\operatorname{Re}} \nabla b_{1}^{K}: \nabla b_{2}^{K} d \Omega \mathbf{I}\right. \\
& \left.+\int_{K} \frac{1}{\operatorname{Re}} \nabla b_{1}^{K} \otimes \nabla b_{2}^{K} d \Omega\right), \\
\mathbf{R} & =\int_{K} b_{2}^{K} \overline{\mathbf{r}} d \Omega,
\end{aligned}
$$

where $\mathbf{I}$ is a nd $\times$ nd identity matrix and both $\nabla b_{1}^{K}$ and $\nabla b_{2}^{K}$ are nd $\times 1$ vectors of gradient of the bubble functions. Substituting (19) into (13), then we can obtain the fine scale $\widehat{\mathbf{u}}$ over the $\Omega^{K}$ as follows:

$$
\left.\widehat{\mathbf{u}}\right|_{K}=b_{1}^{K}(\boldsymbol{\xi}) \boldsymbol{\beta}=b_{1}^{K}(\boldsymbol{\xi}) \mathbf{S}^{-1} \mathbf{R} .
$$

Step 3 (solution of the coarse scale problem $\bar{W}$ ). Once we have obtained the fine scale solution $\widehat{\mathbf{u}}$, now we turn our attention to the coarse scale problem $\bar{W}$. Exploiting the linearity of the solution slot between $\overline{\mathbf{u}}$ and $\widehat{\mathbf{u}},(10)$ can be written as

$$
\begin{aligned}
(\overline{\mathbf{w}}, \dot{\overline{\mathbf{u}}}) & +\left(\overline{\mathbf{w}}, \mathbf{u}^{c} \cdot \nabla \overline{\mathbf{u}}\right)+\left(\overline{\mathbf{w}}, \mathbf{u}^{c} \cdot \nabla \widehat{\mathbf{u}}\right)+\left(\nabla \overline{\mathbf{w}}, \frac{1}{\operatorname{Re}} \nabla \overline{\mathbf{u}}\right) \\
& +\left(\nabla \overline{\mathbf{w}}, \frac{1}{\operatorname{Re}} \nabla \widehat{\mathbf{u}}\right)=0 .
\end{aligned}
$$

Using the integration by parts for the terms containing $\widehat{\mathbf{u}}$ in (22) as well as the assumptions used in (5), (22) has the following forms:

$$
\begin{aligned}
(\overline{\mathbf{w}}, \dot{\overline{\mathbf{u}}})+\left(\overline{\mathbf{w}}, \mathbf{u}^{c} \cdot \nabla \overline{\mathbf{u}}\right)-\left(\mathbf{u}^{c} \cdot \nabla \overline{\mathbf{w}}, \widehat{\mathbf{u}}\right)+\left(\nabla \overline{\mathbf{w}}, \frac{1}{\operatorname{Re}} \nabla \overline{\mathbf{u}}\right) \\
-\left(\nabla \cdot\left(\frac{1}{\operatorname{Re}} \nabla \overline{\mathbf{w}}\right), \widehat{\mathbf{u}}\right)=0 .
\end{aligned}
$$

Substituting (18) into (23), subsequently assuming that the coarse scale residual $\overline{\mathbf{r}}$ is a constant over the $\Omega^{K}$, after rearrangement we get the finally computed formulation:

$$
\begin{aligned}
& (\overline{\mathbf{w}}, \dot{\overline{\mathbf{u}}})+\left(\overline{\mathbf{w}}, \mathbf{u}^{c} \cdot \nabla \overline{\mathbf{u}}\right)+\left(\mathbf{u}^{c} \cdot \nabla \overline{\mathbf{w}}+\nabla\right. \\
& \left.\quad\left(\frac{1}{\operatorname{Re}} \nabla \overline{\mathbf{w}}\right), \boldsymbol{\tau}\left(\dot{\overline{\mathbf{u}}}+\mathbf{u}^{c} \cdot \nabla \overline{\mathbf{u}}-\nabla \cdot\left(\frac{1}{\operatorname{Re}} \nabla \overline{\mathbf{u}}\right)\right)\right)=0,
\end{aligned}
$$

where

$$
\begin{aligned}
\boldsymbol{\tau} & =b_{1}^{K} \int_{K} b_{2}^{K} d \Omega\left[\int_{K} b_{2}^{K} \mathbf{u}^{c} \cdot \nabla b_{1}^{K} d \Omega \mathbf{I}\right. \\
& +\left(\int_{K} \frac{1}{\operatorname{Re}} \nabla b_{1}^{K}: \nabla b_{2}^{K} d \Omega \mathbf{I}+\int_{K} \frac{1}{\operatorname{Re}} \nabla b_{1}^{K}\right. \\
& \left.\left.\otimes \nabla b_{2}^{K} d \Omega\right)\right]^{-1} .
\end{aligned}
$$

Compared with the standard variational form (2), it is important to note that the third term on the left appears in (24) owing to the assumption that there exists fine scale in the problem, which in fact models the effect of the fine scale.
2.2. Low-Pass Filter. Although the VMEFG method can play a role in stability for some moderate Re values [21], but for very large Re values, it will still produce spurious oscillations as we will see in the next section. Previous researches [2224,28 ] indicate that a low-pass filter can suppress the spurious oscillations; thus in the paper we use the high order low-pass filter to smooth the solutions for very large Re values at each time loop.

For one-dimensional problems, a high order tridiagonal filter can be formulated as $[23,24]$

$$
\alpha_{f} \widehat{\phi}_{i-1}+\widehat{\phi}_{i}+\alpha_{f} \widehat{\phi}_{i+1}=\sum_{m=1}^{M} \frac{a_{n}}{2}\left(\phi_{i+m}+\phi_{i-m}\right) \text {, }
$$

where $\phi_{i}$ denotes the given value at point $i$ and $\widehat{\phi}_{i}$ denotes the value after filtering. With proper choices of coefficients, (22) provides a $2 M$ th-order formulation on a $2 M+1$ point stencil. The $M+1$ coefficients $a_{0}, a_{1}, \ldots, a_{M}$ can be obtained in terms of $\alpha_{f}$ by Taylor expansion. In the paper, we use the following coefficients of a family of 10th-order filter derived in [24, 28]

$$
\begin{aligned}
& a_{0}=\frac{193+126 \alpha_{f}}{256}, \\
& a_{1}=\frac{105+302 \alpha_{f}}{256}, \\
& a_{2}=\frac{15\left(-1+\alpha_{f}\right)}{64}, \\
& a_{3}=\frac{45\left(1-2 \alpha_{f}\right)}{512}, \\
& a_{4}=\frac{5\left(-1+2 \alpha_{f}\right)}{256}, \\
& a_{5}=\frac{1-2 \alpha_{f}}{512} .
\end{aligned}
$$

For two-dimensional problems, the explicit high order filter can refer to [29].

\section{Numerical Examples}

To show the effectiveness of the combination VMEFG and low-pass filter, numerical solutions of Burgers' equation with small diffusivity coefficients for three problems will be considered.

Example 1. Consider the following 1D Burgers' problem:

$$
\frac{\partial u}{\partial t}+u \frac{\partial u}{\partial x}=\frac{1}{R e} \frac{\partial^{2} u}{\partial x^{2}}, \quad 0<x<1, t>0,
$$

with Dirichlet boundary conditions

$$
\begin{aligned}
& u(0, t)=u_{0}, \\
& u(1, t)=\bar{u},
\end{aligned}
$$




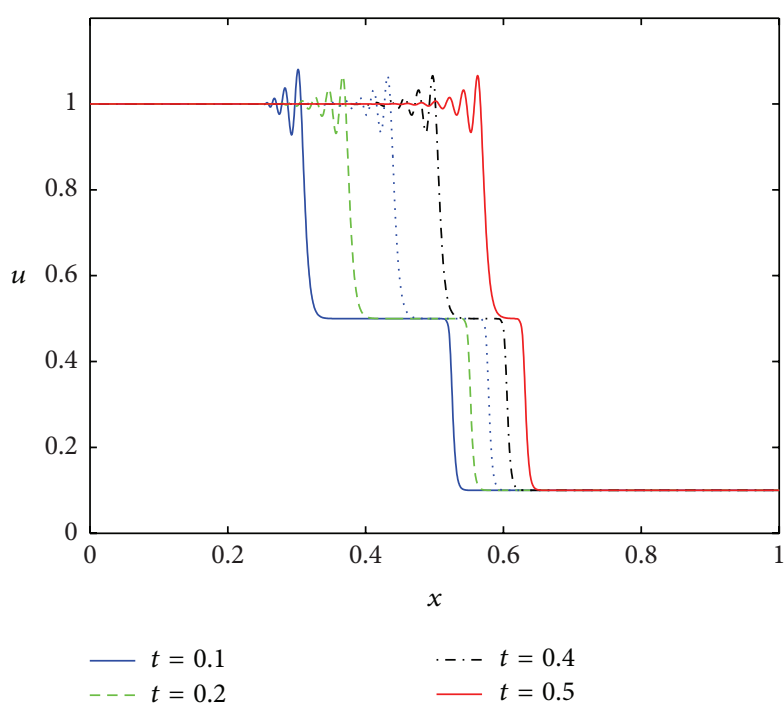

(a)

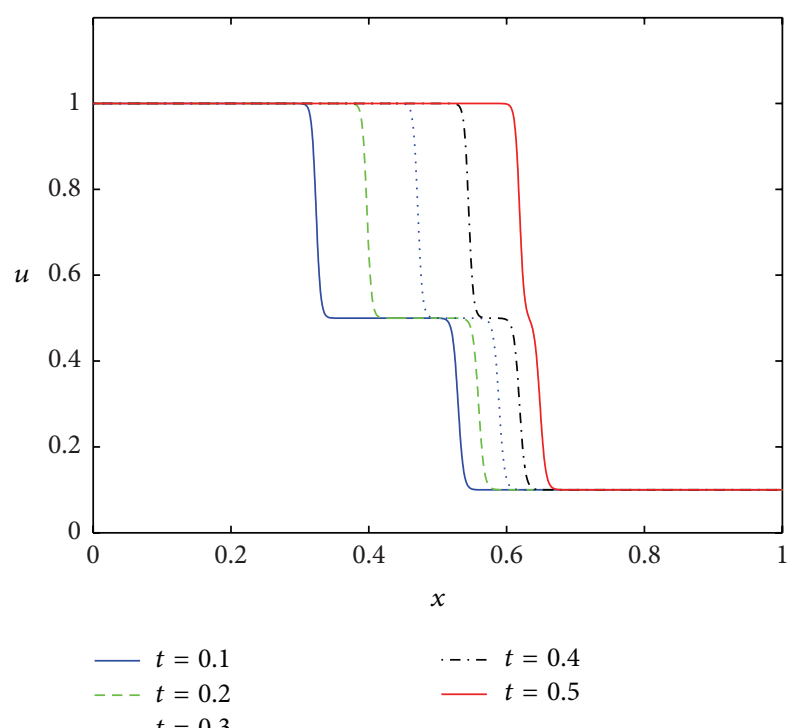

(b)

FIGURE 2: The numerical solutions of $\mathrm{Re}=2000$ obtained by VMEFG method with and without filter, respectively. (a) is obtained without filter and (b) is with low-pass filter.

The exact solution of the problem is given by $[10,11]$

$$
u(x, t)=\frac{0.1 e^{-A}+0.5 e^{-B}+e^{-C}}{e^{-A}+e^{-B}+e^{-C}},
$$

where

$$
\begin{aligned}
& A=0.05 \operatorname{Re}(x-0.5+4.95 t), \\
& B=0.25 \operatorname{Re}(x-0.5+0.75 t), \\
& C=0.5 \operatorname{Re}(x-0.375) .
\end{aligned}
$$

For this problem, extensive numerical results indicated that the solutions are smooth when $\operatorname{Re} \leq 200$ [11]; as the $\operatorname{Re}$ number increases, the solutions will appear discontinuous.

Figure 2 shows the VMEFG solutions without filter and with filter at different times and $\mathrm{Re}=2000$. It can be seen that VMEFG solutions have some spurious oscillation under this larger Re number, while the solutions obtained by VMEFG method with filter are still smooth. This example also illustrates that VMEFG method produces the numerical pseudo oscillations for very small diffusivity coefficient. Thus, other techniques need to overcome the difficulty.

Figure 3 presents the comparison of solutions obtained by VMEFG method with filter and exact solutions at different times. It can be seen that the VMEFG method with filter solutions are in well agreement with the exact solutions. From Figures 2 and 3, it can be seen that the VMEFG method coupled high order low-pass filter is one of the techniques which can work out the difficulty well.

In order to investigate the accuracy of the VMEFG method with filter, the following $L_{2}$ norm error is used:

$$
\|e\|_{2}=\left(h \sum_{i=1}^{N}\left(u_{i}^{\text {num }}-u_{i}^{\text {exact }}\right)^{2}\right)^{1 / 2}
$$

TABLE 1: The $L_{2}$ errors obtained by VMEFG method with filter at $t=0.1,0.3$, and 0.5 .

\begin{tabular}{lccc}
\hline$h$ & $t=0.1$ & $t=0.3$ & $t=0.5$ \\
\hline $1 / 200$ & 0.032125 & 0.046050 & 0.064297 \\
$1 / 400$ & 0.016440 & 0.024454 & 0.034351 \\
$1 / 800$ & 0.009747 & 0.015153 & 0.020432 \\
$1 / 1600$ & 0.004423 & 0.006895 & 0.009768 \\
\hline
\end{tabular}

where $h$ is the space step, $N$ is the number of node, $u^{\text {num }}$ is the numerical solution, and $u^{\text {exact }}$ is the exact solution. Table 1 shows the $L_{2}$ norm errors by taking different numbers of node at different times for the simulation and observes that the numerical results are satisfactory. Figure 4 illustrates these errors plotted against $h$ on a log-log scale. It can be noted that the order of convergence of the VMEFG method with filter is linear.

Example 2. In this example, we consider the 1D Burgers' equation

$$
\frac{\partial u}{\partial t}+u \frac{\partial u}{\partial x}=\frac{1}{\operatorname{Re}} \frac{\partial^{2} u}{\partial x^{2}}, \quad 0<x<1, t>0
$$

with the initial condition

$$
u(x, 0)=\sin (\pi x), \quad 0<x<1,
$$

and the boundary conditions

$$
u(0, t)=u(1, t)=0.0, \quad t>0 .
$$




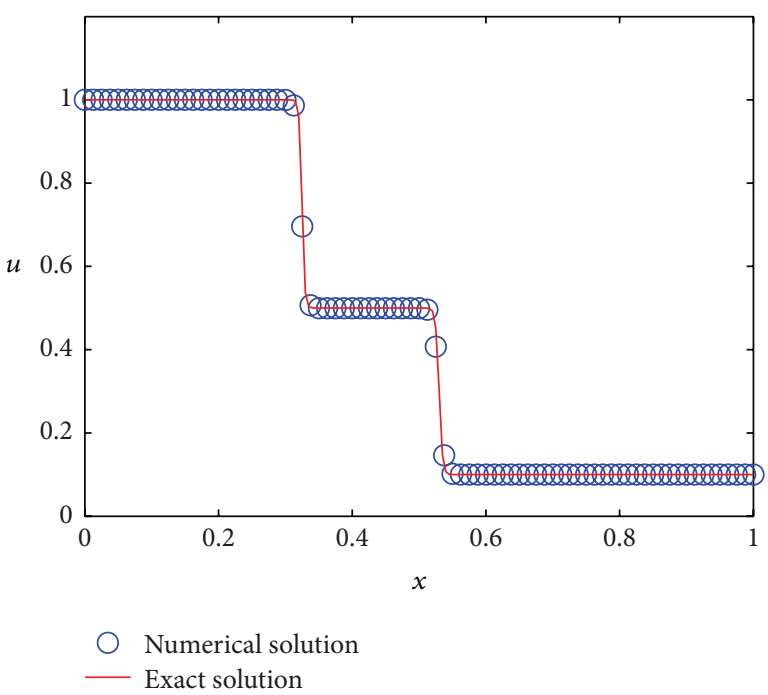

(a) $t=0.1$

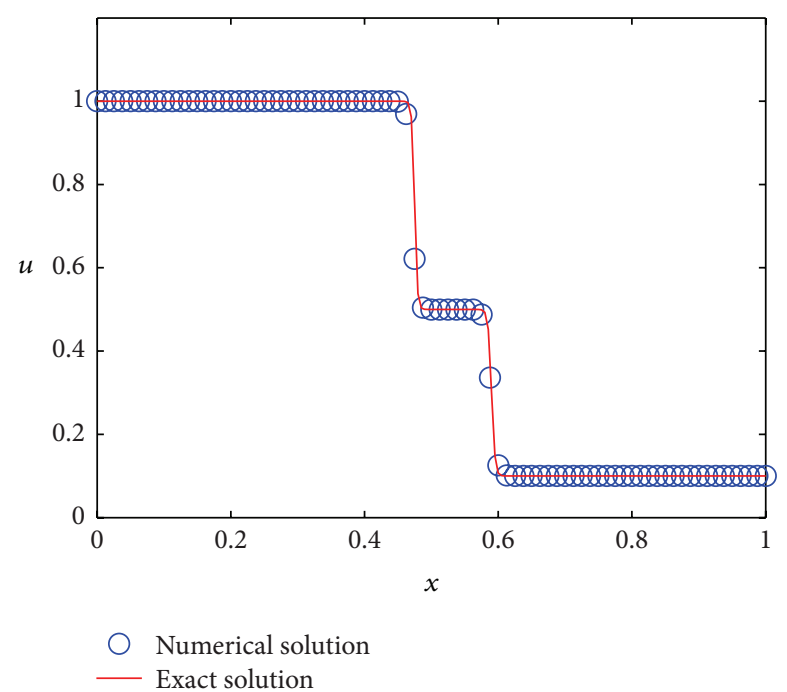

(b) $t=0.3$

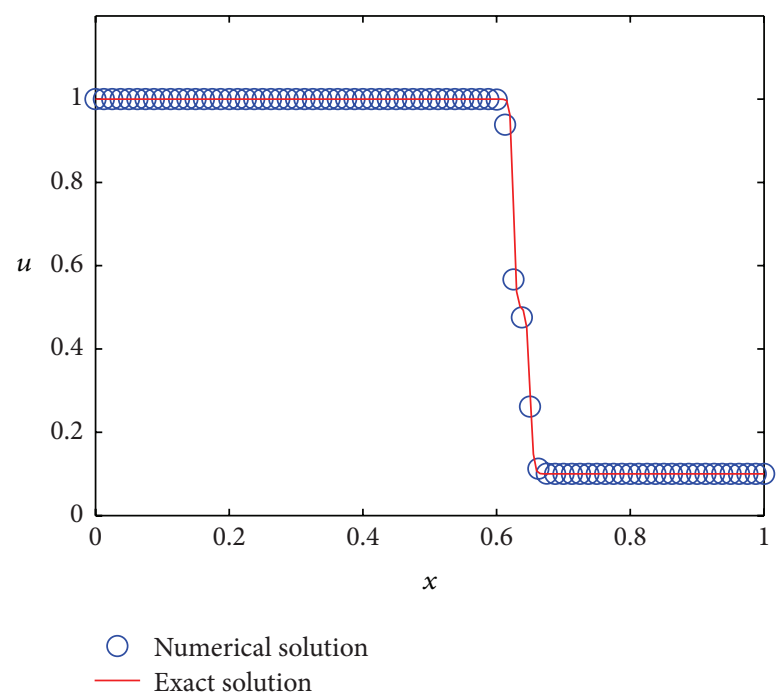

(c) $t=0.5$

FIGURE 3: Comparison of VMEFG method with filter and exact solution at $t=0.1,0.3$, and 0.5 .

The exact solution of this problem is $[10,30]$

$$
\begin{aligned}
& u(x, t) \\
& \quad=2 \pi \frac{1}{\operatorname{Re}} \frac{\sum_{n=1}^{\infty} a_{n} \exp \left(-n^{2} \pi^{2} t / \mathrm{Re}\right) n \sin (n \pi x)}{a_{0}+\sum_{n=1}^{\infty} a_{n} \exp \left(-n^{2} \pi^{2} t / \mathrm{Re}\right) \cos (n \pi x)},
\end{aligned}
$$

where the Fourier coefficients are

$$
\begin{aligned}
& a_{0}=\int_{0}^{1} \exp \left\{-\frac{\mathrm{Re}}{2 \pi}[1-\cos (\pi x)]\right\} d x, \\
& a_{n}=\int_{0}^{1} \exp \left\{-\frac{\mathrm{Re}}{2 \pi}[1-\cos (\pi x)]\right\} \cos (n \pi x) d x \\
& \quad(n=1,2,3, \ldots) .
\end{aligned}
$$

Although the example has the exact solution, the convergence is very slow for larger value of Re owing to that the exact solution of the equation involves Fourier series solutions. Therefore, in order to show the computational accuracy of the proposed method for small diffusivity coefficients, that is, large Re values, the numerical solutions are compared with the results available in the literatures $[3,30]$.

In this example, 200 nodes are distributed uniformly in the domain and $\Delta t=0.001$. Figure 5 presents the numerical solutions obtained by EFG method and VMEFG method for $\operatorname{Re}=10^{3}$. It can be seen that there exists some oscillation in the solution of EFG, but, in contrast, the solutions of VMEFG are always smooth, which indicates that VMEFG method has the ability to accommodate the exact solution to the problem. Meanwhile, this demonstrates that the VMEFG has better stability and accuracy than EFG method for moderate large Re number indeed. 


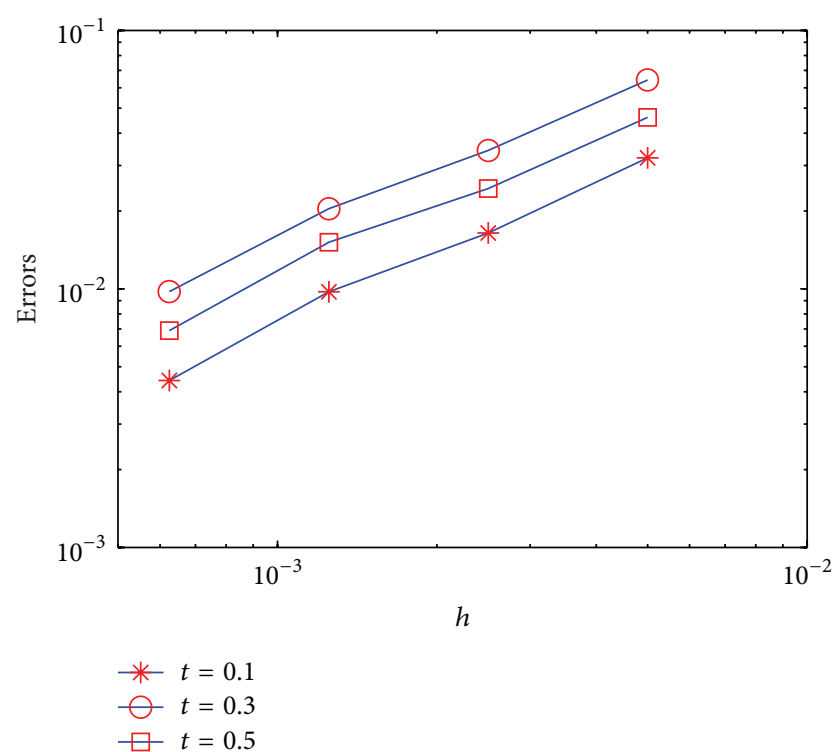

FIGURE 4: The errors from Table 1 plotted against $h$ on a log-log scale.

Figure 6 illustrates the numerical results obtained by VMEFG without and with low-pass filter at different time for $\operatorname{Re}=10^{4}$. It can be observed that the profiles of $u$ develop extremely sharp gradients near the right-hand boundary for $t \geq 0.4$, and the numerical results of VMEFG method without filter show the nonphysical oscillations near the righthand boundary for $t \geq 0.6$, which indicates that the VMEFG method still has spurious oscillations for very large Re numbers, whereas the numerical results obtained by VMEFG method with low-pass filter are still smooth and meanwhile the sharp gradients of the profiles near $x=1$, especially for $t=0.6$, are well distinguished.

Table 2 shows the results obtained by VMEFG method with low-pass filter at $t=1.0$ for $\operatorname{Re}=10^{4}$ and those available in the literatures. It can be found that our results are in agreement with the results presented in [30] for $\mathrm{Re}=10^{4}$. From Figure 6 and Table 2, we can conclude that the VMEFG method with low-pass filter gives remarkable accuracy for large values of Re.

The numerical solution of Burgers' equation at a high Reynolds number ( $\operatorname{Re} \geq 10^{5}$ ) is very difficult due to the presence of shock [21]. A direct application of the VMEFG method for $\mathrm{Re}=10^{5}$ leads to highly oscillatory results as shown in Figure 7. It can be seen that the oscillation starts at $t=0.4$ and is accumulated and amplified in the following times. Figure 7 also shows the results obtained by using the low-pass filter under the same condition. It can be found that the oscillations are eliminated and sharp shock profiles are resolved. Meanwhile, we consider $\operatorname{Re}=10^{9}$ further. Figure 8 shows the results obtained without and with low-pass filter for $\operatorname{Re}=10^{9}$. Figure $8(\mathrm{a})$ is obtained without filter; we see some numerical oscillations obviously. But the low-pass filter eliminates those high frequency oscillations very well.

Example 3. For the third example, we consider the coupled nonlinear system of Burgers' equation which has been studied
TABLE 2: Comparison between the numerical solutions at $t=1.0$ for $\operatorname{Re}=10^{4}$.

\begin{tabular}{lccc}
\hline$x$ & Reference [3] & Reference [30] & VMEFG with filter \\
\hline 0.05 & 0.0424 & 0.0379 & 0.037716792 \\
0.11 & 0.0843 & 0.0834 & 0.082959609 \\
0.16 & 0.1263 & 0.1213 & 0.120632606 \\
0.22 & 0.1684 & 0.1667 & 0.165785694 \\
0.27 & 0.2103 & 0.2044 & 0.203352212 \\
0.33 & 0.2522 & 0.2497 & 0.248337909 \\
0.38 & 0.2939 & 0.2872 & 0.285730246 \\
0.44 & 0.3355 & 0.3322 & 0.330462517 \\
0.50 & 0.3769 & 0.3769 & 0.375015652 \\
0.55 & 0.4182 & 0.4141 & 0.411982983 \\
0.61 & 0.4592 & 0.4584 & 0.456120122 \\
0.66 & 0.4999 & 0.4951 & 0.492687106 \\
0.72 & 0.5404 & 0.5388 & 0.536270966 \\
0.77 & 0.5805 & 0.5749 & 0.572307656 \\
0.83 & 0.620 & 0.6179 & 0.615158458 \\
0.88 & 0.6600 & 0.6533 & 0.650490215 \\
0.94 & 0.6957 & 0.6952 & 0.692359873 \\
0.96 & - & 0.7090 & 0.706171615 \\
0.98 & - & 0.7228 & 0.719903495 \\
0.99 & - & 0.7296 & 0.728178293 \\
\hline
\end{tabular}

by Zhang et al. [14] and Donea et al. [7]. The coupled nonlinear system of Burgers' equation is considered over the square domain $\Omega=[0,1] \times[0,1]$ and defined as the following:

$$
\begin{gathered}
\frac{\partial u}{\partial t}+u \frac{\partial u}{\partial x}+v \frac{\partial u}{\partial y}=\frac{1}{\operatorname{Re}}\left(\frac{\partial^{2} u}{\partial x^{2}}+\frac{\partial^{2} u}{\partial y^{2}}\right), \\
\frac{\partial v}{\partial t}+u \frac{\partial v}{\partial x}+v \frac{\partial v}{\partial y}=\frac{1}{\operatorname{Re}}\left(\frac{\partial^{2} v}{\partial x^{2}}+\frac{\partial^{2} v}{\partial y^{2}}\right),
\end{gathered}
$$

with the initial conditions

$$
\begin{array}{ll}
u(x, y, 0)=\sin (\pi x) \cos (\pi y), & (x, y) \in \Omega, \\
v(x, y, 0)=\cos (\pi x) \sin (\pi y), & (x, y) \in \Omega,
\end{array}
$$

and the boundary conditions

$$
\begin{gathered}
u(0, y, t)=u(1, y, t)=0.0, \quad t>0, \\
v(x, 0, t)=v(x, 1, t)=0.0 \quad t>0, \\
\frac{\partial u}{\partial n}(x, 0, t)=\frac{\partial u}{\partial n}(x, 1, t)=0.0 \quad t>0, \\
\frac{\partial v}{\partial n}(0, y, t)=\frac{\partial v}{\partial n}(1, y, t)=0.0 \quad t>0 .
\end{gathered}
$$

The above problem has various symmetries. As Zhang et al. [14] pointed out, there exist $u(x, y, t)=v(y, x, t)$ and $u(x, y, t)=-u(1-x, 1-y, t)$ over the global domain and $u(x, x, t)=v(x, x, t)$ and $u(x, x, t)=-u(1-x, 1-x, t)$ along the transverse section of the domain. Thus, in the following, we only present the results of $u(x, y, t)$. 


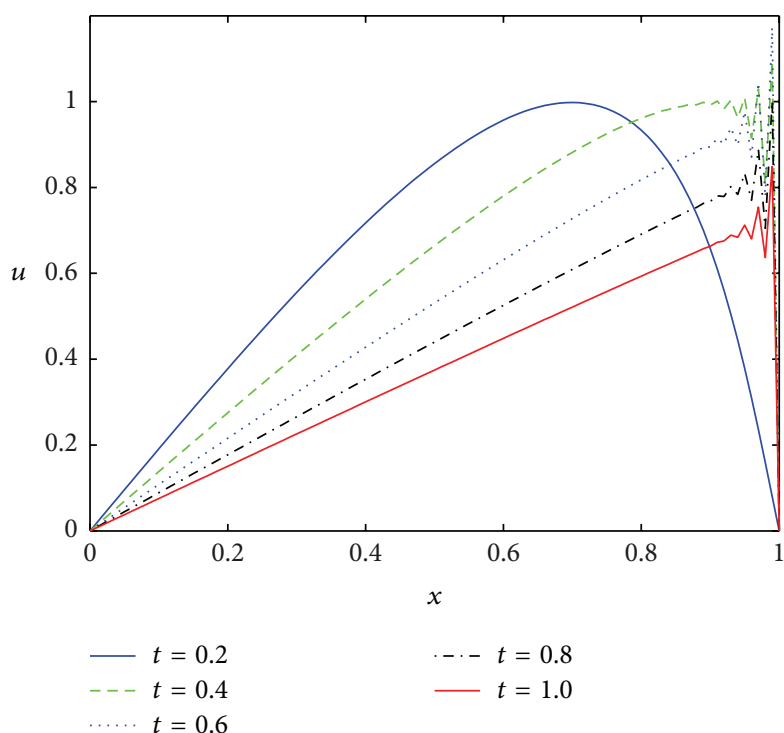

(a)

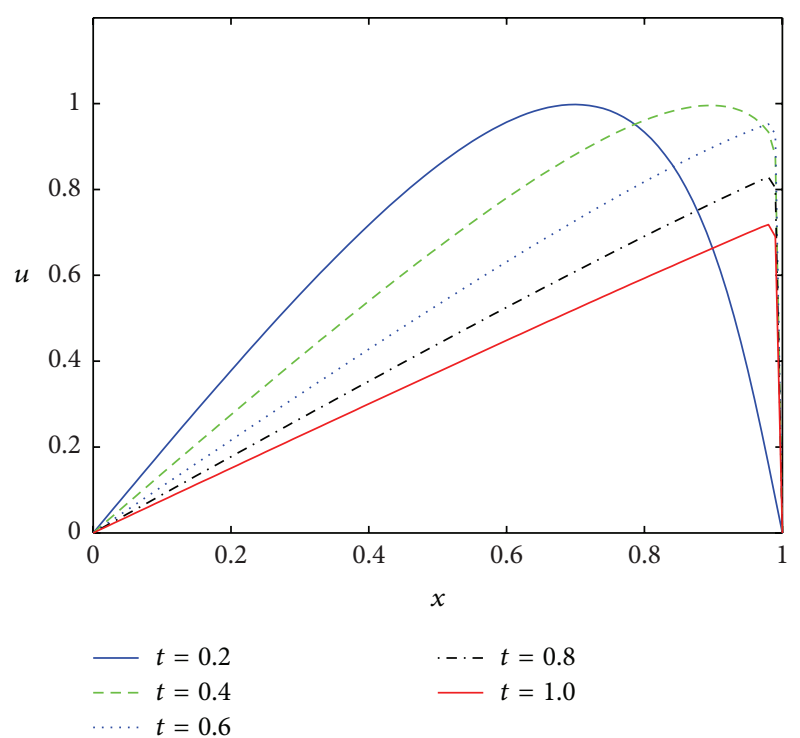

(b)

FIGURE 5: The numerical solutions of Re $=10^{3}$ via EFG and VMEFG, respectively. (a) is obtained by EFG and (b) is obtained by VMEFG.

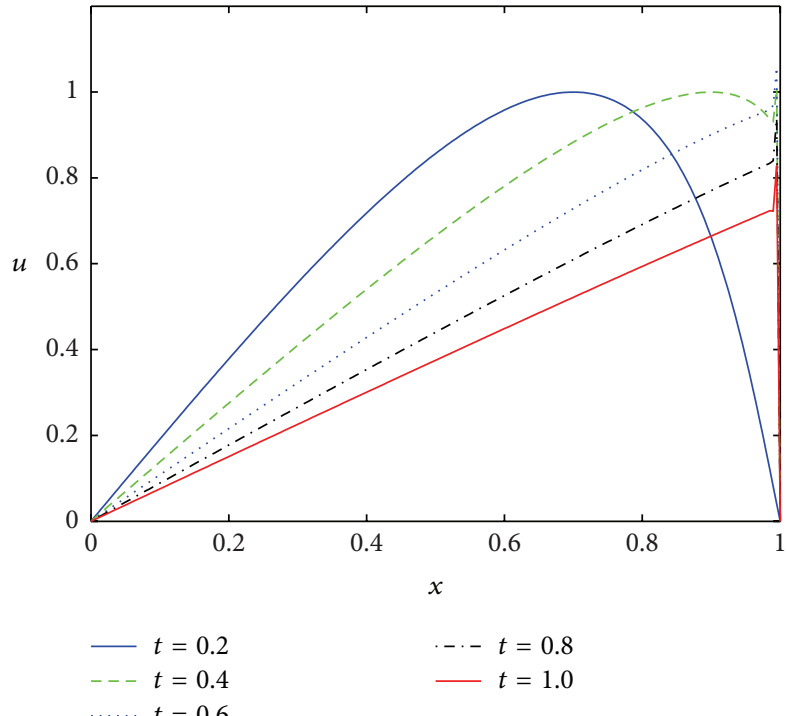

(a)

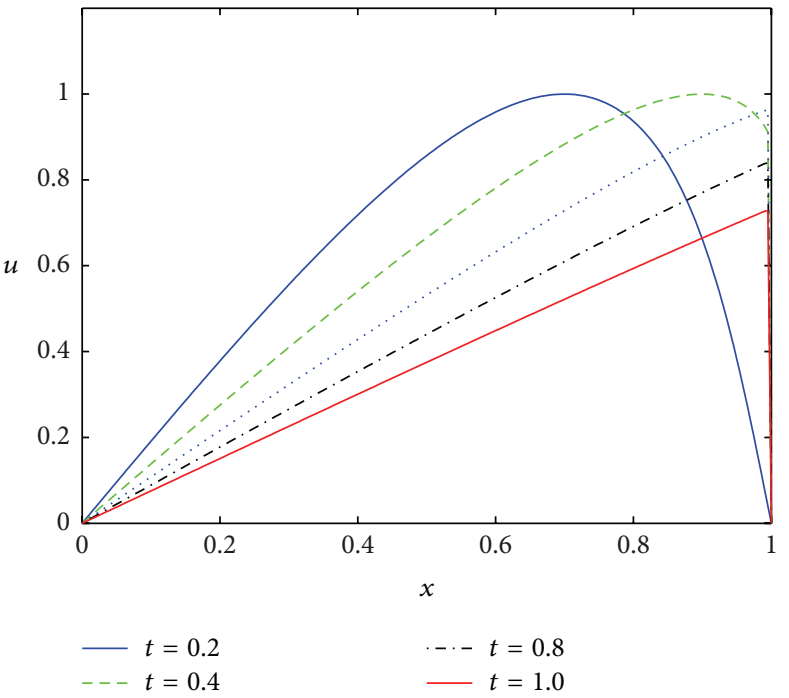

(b)

FIGURE 6: Effects of filtering for solving one-dimensional Burgers' equation for $\mathrm{Re}=10^{4}$ by the VMEFG method. (a) is obtained without filter and (b) is with low-pass filter.

This example was solved by many researchers and in their studies, the maximum value of Re is the order of $10^{3}$. Meanwhile, from those results available in the literature [14], we can see that spurious oscillation still appeared for $\operatorname{Re}=10^{3}$.

In this study, $51 \times 51$ nodes are distributed uniformly in the domain and $\Delta t=0.01$. Figure 9 illustrates the comparison for effects of low-pass filter. It can be seen that the spurious oscillation also exists in our results obtained by using VMEFG method without filter, whereas the results obtained with lowpass filter are excellent; clearly, all oscillations are effectively removed and the shock front is very sharp. To quantify the computational accuracy and stabilization of VMEFG with low-pass filter, the results of $u$ along the domain diagonal ( $y=$ $x$ ) are presented in Figure 10. It can be seen obviously that the results obtained by VMEFG have some spurious oscillation, while the results obtained by VMEFG with low-pass filter have no spurious oscillation and the sharp shock profiles are resolved.

To test the VMEFG method with low-pass filter for solving this coupled Burgers' equataion further, we next consider 




(a)

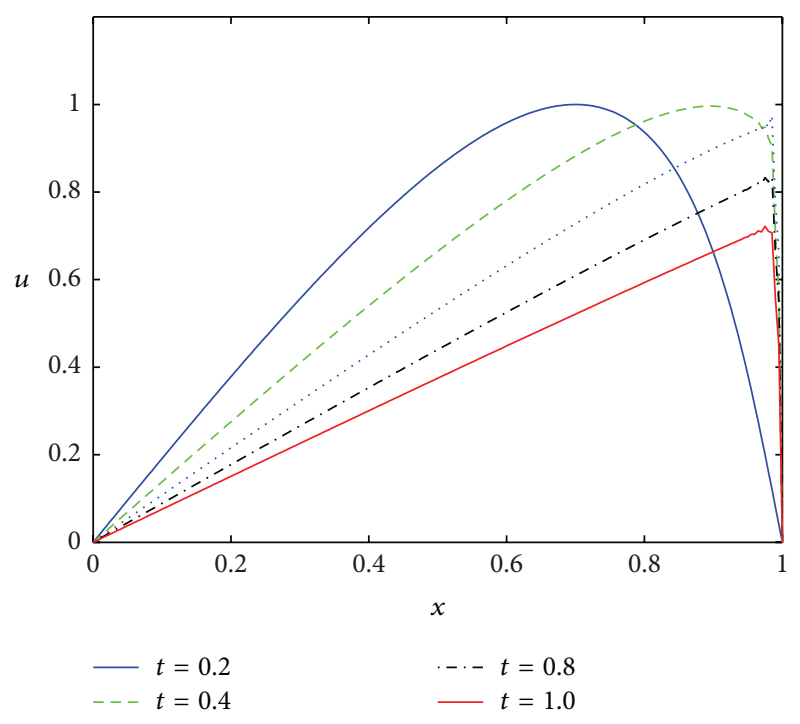

(b)

FIGURE 7: Effects of filtering for solving one-dimensional Burgers' equation for $\mathrm{Re}=10^{5}$ by the VMEFG method. (a) is obtained without filter and (b) is with low-pass filter.

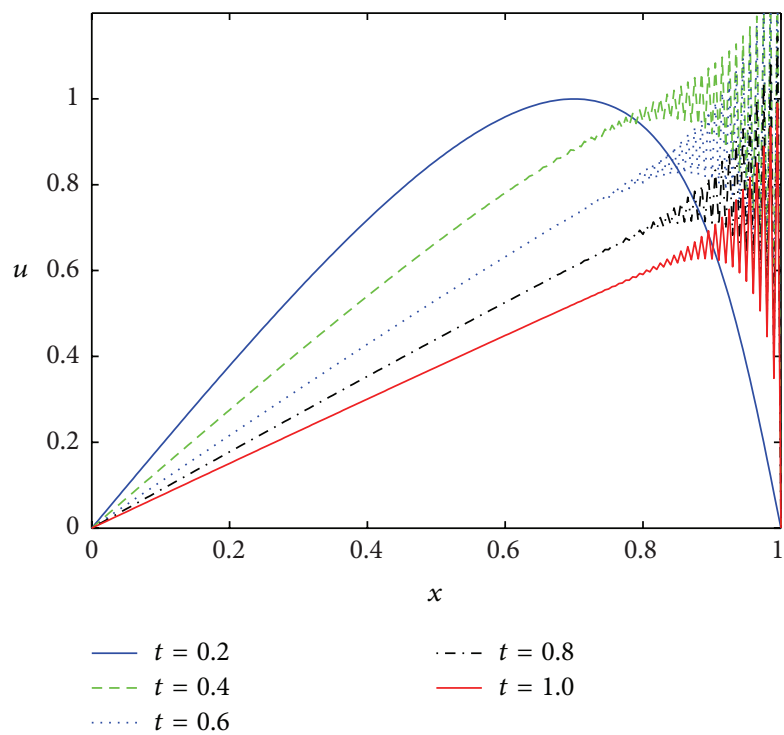

(a)



(b)

FIGURE 8: Effects of filtering for solving one-dimensional Burgers' equation for $\operatorname{Re}=10^{9}$ by the VMEFG method. (a) is obtained without filter and (b) is with low-pass filter.

the $\mathrm{Re}=10^{4}$ and the results are presented in Figure 11. To the best of our knowledge, there have been no results reported on so large Re value. The top four figuers are obtained without filter; we see that some numerical oscillations appear along the diagnonal of the domain passing through the points $(0,1)$ and $(1,0)$. But the low-pass filter eliminates those very well and the shocks along the diagonal of the domain are also very sharp. Figure 12 shows the results obtained by VMEFG with low-pass filter along the domain diagonal $(y=x)$ for $\operatorname{Re}=10^{4}$. It can be noted obviously that the solutions are also smooth and the sharp shock profiles are distinguished.

\section{Conclusions}

In this paper, VMEFG method in conjunction with lowpass filter has been applied for the numerical solution of 

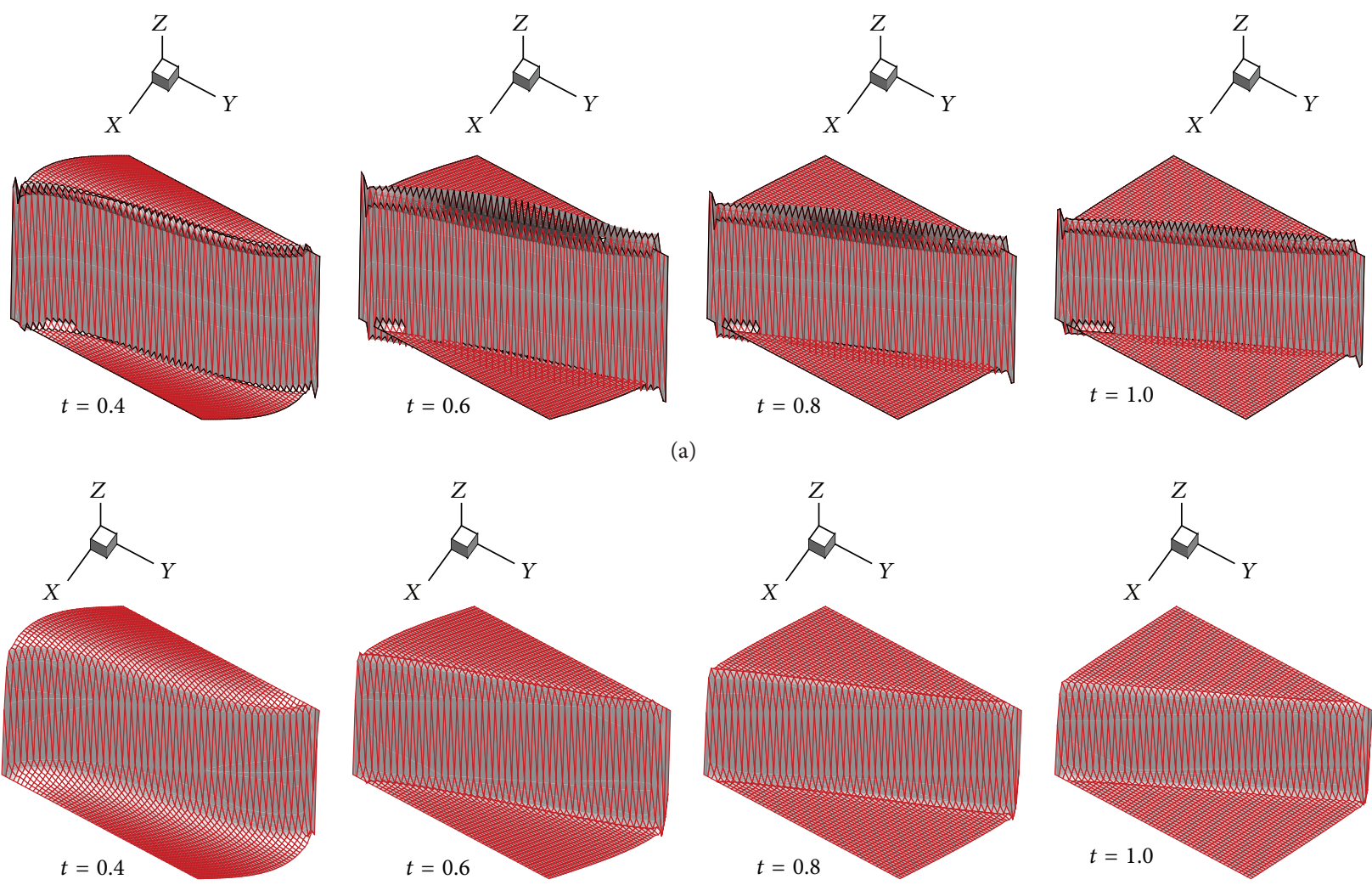

(b)

Figure 9: 2D Burgers' equation for $\mathrm{Re}=10^{3}$. (a) are computed without filter; (b) are with the low-pass filter.

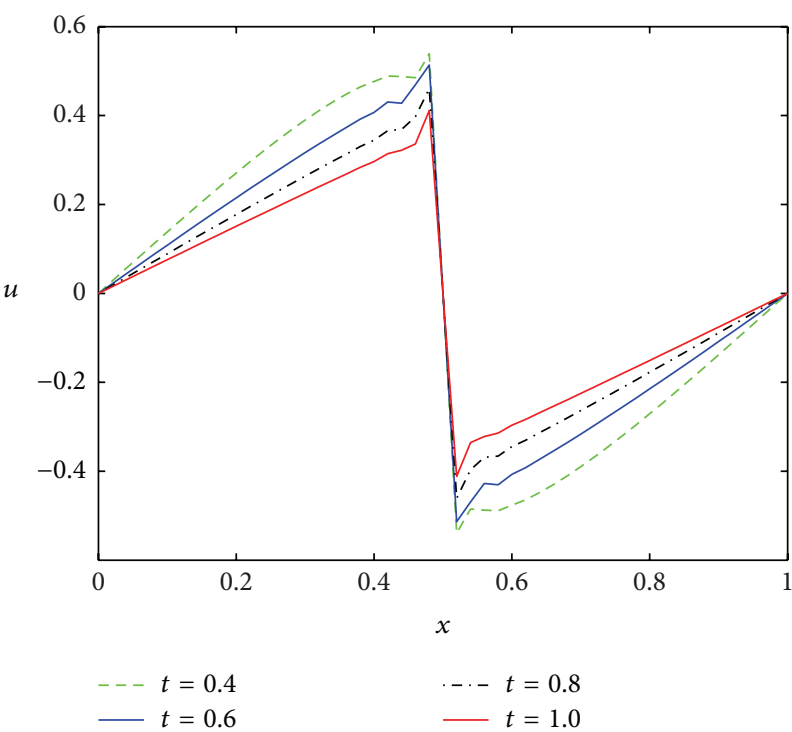

(a)

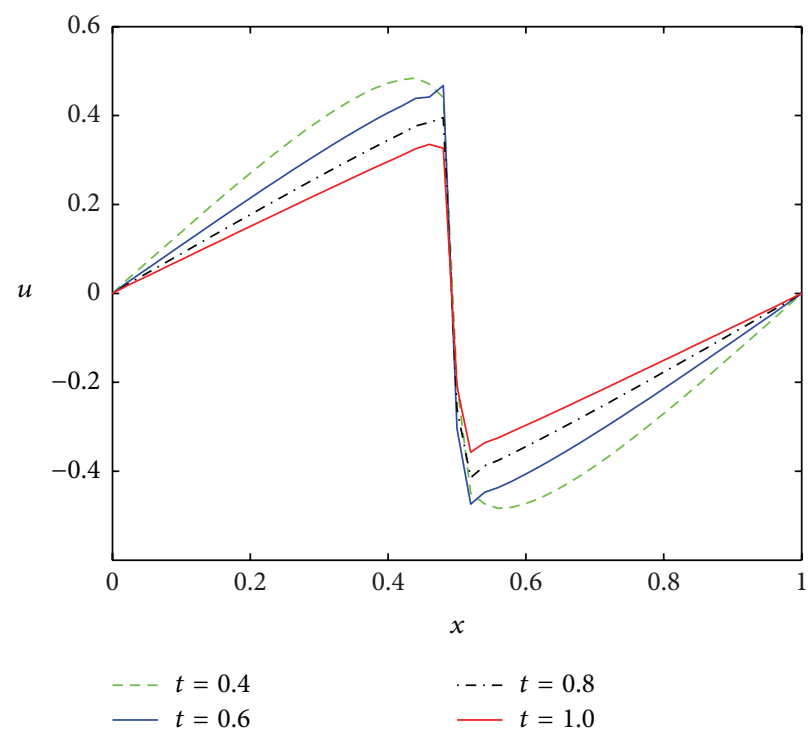

(b)

FIgURE 10: Solutions $u(x, x, t)$ of the VMEFG wihout filter (a) and with low-pass filter (b) at different times for $\operatorname{Re}=10^{3}$.

Burgers' equations with small diffusivity coefficients, which lead to convection dominated problem. To the best of our knowledge, this is the first time for meshless methods to be used for solving Burgers' equation with such small diffusivity coefficients. The accuracy and stabilization of the proposed method are validated by three numerical examples. Compared with numerical results available in the literatures, it can be seen that the VMEFG method coupled with low-pass 

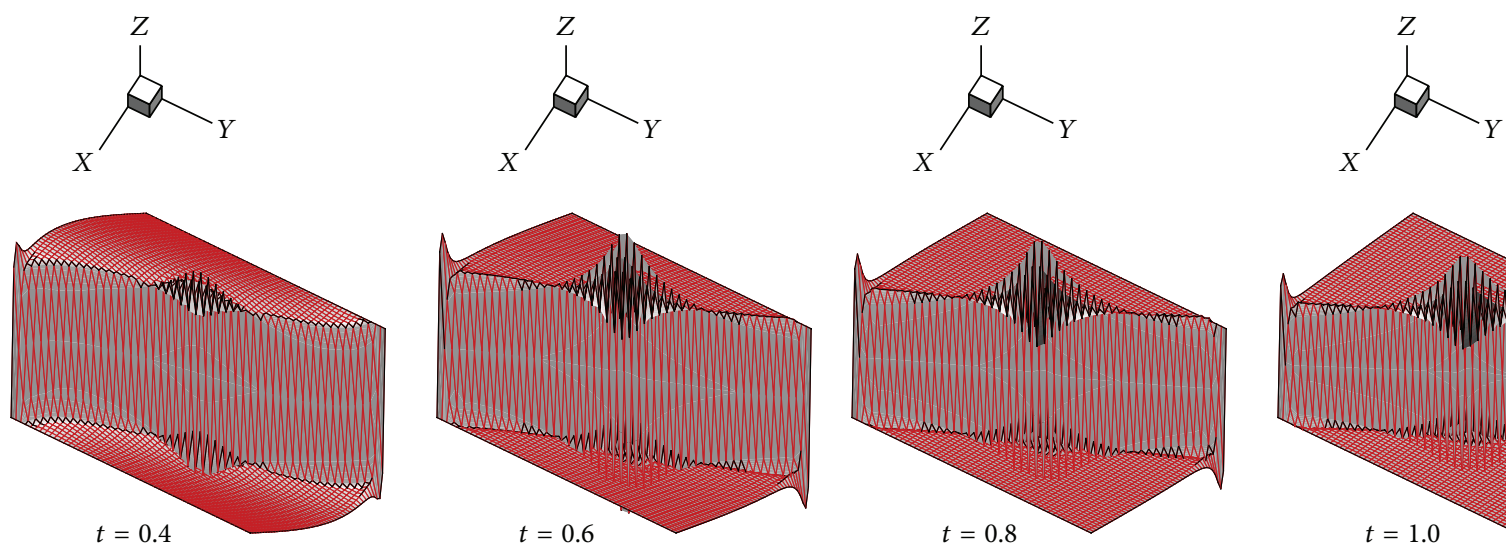

(a)
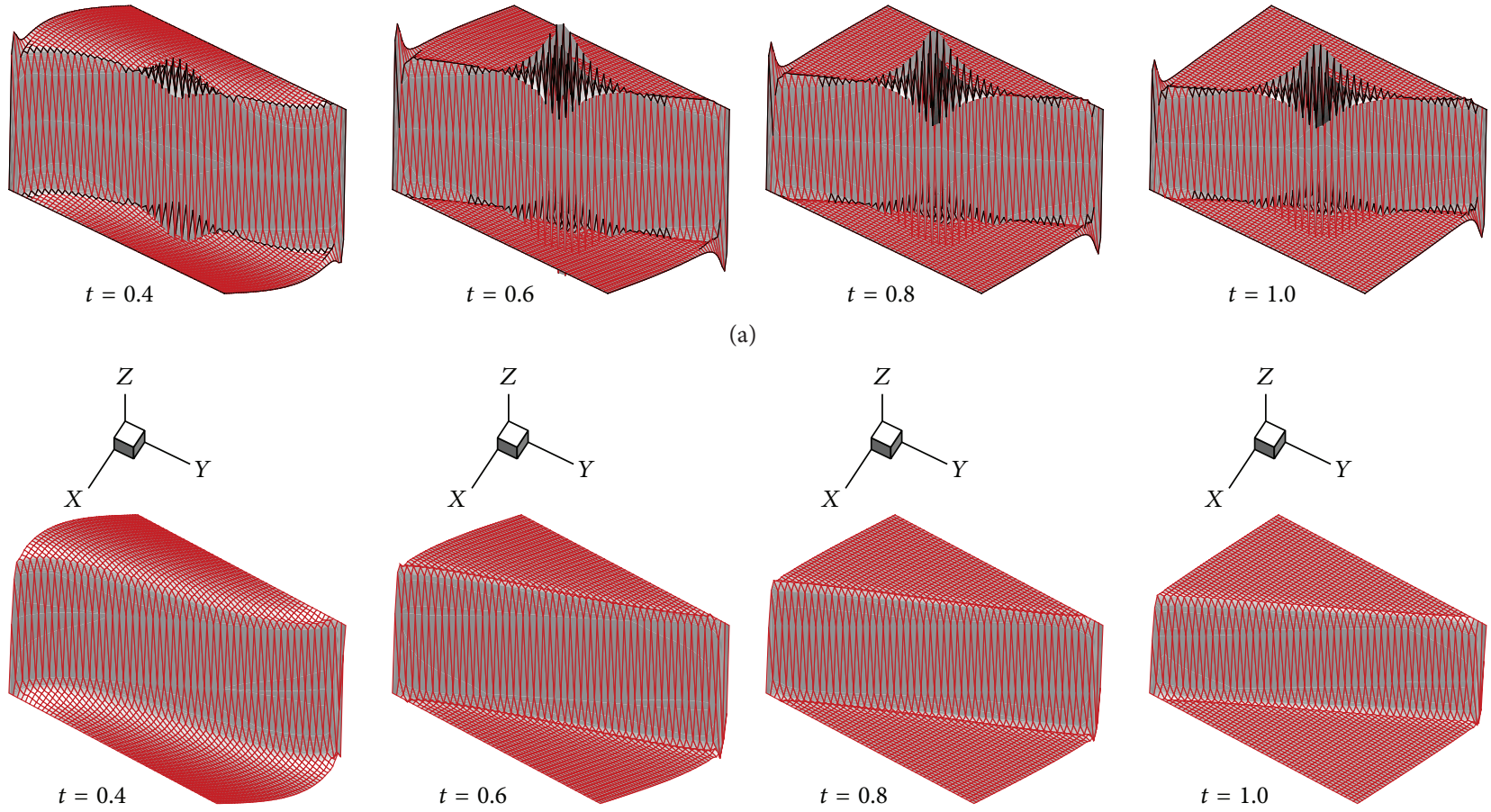

(b)

FIGURE 11: 2D Burgers' equation for Re $=10^{4}$. (a) are computed without filter; (b) are with the low-pass filter.

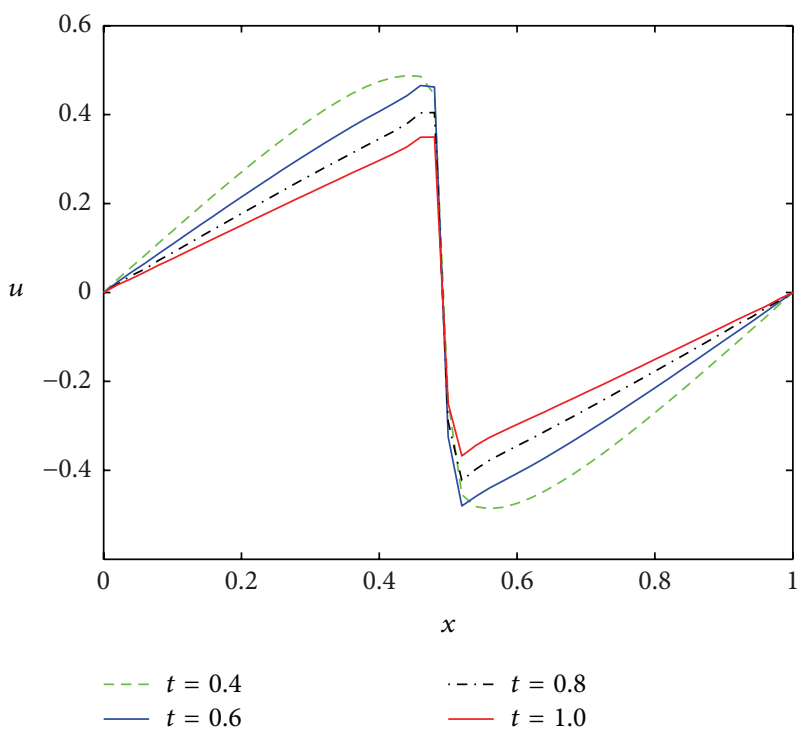

FIGURE 12: Solutions $u(x, x, t)$ of the VMEFG with low-pass filter at different times for $\operatorname{Re}=10^{4}$.

filter produces better results with ease of implementation for Burgers' equation with very large Re numbers.

\section{Conflict of Interests}

The authors declare that there is no conflict of interests regarding the publication of this paper.

\section{Acknowledgments}

This work is financially supported by the Natural Sciences Foundation of China (nos. 11102101, 11171181, and 61273183) and the Academic Mainstay Foundation of Hubei Province of China (no. Q20111208).

\section{References}

[1] P.-G. Zhang and J.-P. Wang, "A predictor-corrector compact finite difference scheme for Burgers' equation," Applied Mathematics and Computation, vol. 219, no. 3, pp. 892-898, 2012.

[2] M. Yousuf, "On the class of high order time stepping schemes based on Padé approximations for the numerical solution of Burgers' equation," Applied Mathematics and Computation, vol. 205, no. 1, pp. 442-453, 2008.

[3] Y. C. Hon and X. Z. Mao, "An efficient numerical scheme for Burgers' equation," Applied Mathematics and Computation, vol. 95, no. 1, pp. 37-50, 1998. 
[4] T. Özis, E. N. Aksan, and A. Özdes, "A finite element approach for solution of Burgers' equation," Applied Mathematics and Computation, vol. 139, pp. 417-428, 2003.

[5] A. Dogan, "A Galerkin finite element approach to Burgers' equation," Applied Mathematics and Computation, vol. 157, no. 2, pp. 331-346, 2004.

[6] A. Huerta, B. Roig, and J. Donea, "Time-accurate solution of stabilized convection-diffusion-reaction equations: II-accuracy analysis and examples," Communications in Numerical Methods in Engineering, vol. 18, no. 8, pp. 575-584, 2002.

[7] J. Donea, B. Roig, and A. Huerta, "High-order accurate timestepping schemes for convection-diffusion problems," Computer Methods in Applied Mechanics and Engineering, vol. 182, no. 3-4, pp. 249-275, 2000.

[8] Z. Guo-Zhong, Y. Xi-Jun, and W. Di, "Numerical solution of the Burgers' equation by local discontinuous Galerkin method," Applied Mathematics and Computation, vol. 216, no. 12, pp. 3671-3679, 2010.

[9] G. Arora and B. K. Singh, "Numerical solution of Burgers' equation with modified cubic B-Spline differential quadrature method," Applied Mathematics and Computation, vol. 224, pp. 166-177, 2013.

[10] S. Dhawan, S. Kapoor, S. Kumar, and S. Rawat, "Contemporary review of techniques for the solution of nonlinear Burgers equation," Journal of Computational Science, vol. 3, no. 5, pp. 405-419, 2012.

[11] S. Kapoor and S. Dhawan, "A computational technique for the solution of Burgers' equation," International Journal of Applied Mathematics and Mechanics, vol. 6, pp. 84-95, 2008.

[12] Y. Gao, L.-H. Le, and B.-C. Shi, "Numerical solution of Burgers' equation by lattice Boltzmann method," Applied Mathematics and Computation, vol. 219, no. 14, pp. 7685-7692, 2013.

[13] A. Huerta and S. F. Méndez, "Time accurate consistently stabilized mesh-free methods for convection dominated problems," International Journal for Numerical Methods in Engineering, vol. 56, no. 9, pp. 1225-1242, 2003.

[14] X. H. Zhang, J. Ouyang, and L. Zhang, "Element-free characteristic Galerkin method for Burgers' equation," Engineering Analysis with Boundary Elements, vol. 33, no. 3, pp. 356-362, 2009.

[15] Y. T. Gu and G. R. Liu, "Meshless techniques for convection dominated problems," Computational Mechanics, vol. 38, no. 2, pp. 171-182, 2006.

[16] T. P. Fries and H. G. Matthies, "A stabilized and coupled meshfree/meshbased method for the incompressible NavierStokes equations-part I: stabilization," Computer Methods in Applied Mechanics and Engineering, vol. 195, no. 44-47, pp. 6205-6224, 2006.

[17] T. P. Fries and H. G. Matthies, "A stabilized and coupled meshfree/meshbased method for the incompressible Navier-Stokes equations-part II: coupling," Computer Methods in Applied Mechanics and Engineering, vol. 195, pp. 6191-6204, 2006.

[18] L. Zhang, J. Ouyang, X. H. Zhang, and W. Zhang, "On a multiscale element-free Galerkin method for the Stokes problem," Applied Mathematics and Computation, vol. 203, no. 2, pp. 745753, 2008.

[19] L. Zhang, J. Ouyang, T. Jiang, and C. L. Ruan, "Variational multiscale element free Galerkin method for the water wave problems," Journal of Computational Physics, vol. 230, no. 12, pp. 5045-5060, 2011.
[20] L. Zhang, J. Ouyang, and X. H. Zhang, "The variational multisclae element free Galerkin method for MHD flows at high Hartmann numbers," Computer Physics Communications, vol. 184, pp. 1106-1118, 2013.

[21] X. H. Zhang and H. Xiang, "Variational multiscale element free Galerkin method for convection-diffusion-reaction equation with small diffusion," Engineering Analysis with Boundary Elements, vol. 46, pp. 85-92, 2014.

[22] G. W. Wei and Y. Gu, "Conjugate filter approach for solving Burgers' equation," Journal of Computational and Applied Mathematics, vol. 149, no. 2, pp. 439-456, 2002.

[23] Y. C. Zhou and G. W. Wei, "High resolution conjugate filters for the simulation of flows," Journal of Computational Physics, vol. 189, no. 1, pp. 159-179, 2003.

[24] J. Li and M. R. Visbal, "High-order compact schemes for nonlinear dispersive waves," Journal of Scientific Computing, vol. 26, no. 1, pp. 1-23, 2006.

[25] V. P. Nguyen, T. Rabczuk, S. Bordas, and M. Duflot, "Meshless methods: a review and computer implementation aspects," Mathematics and Computers in Simulation, vol. 79, no. 3, pp. 763-813, 2008.

[26] T. J. Hughes, G. R. Feijoo, L. Mazzei, and J.-B. Quincy, “The variational multiscale method-a paradigm for computational mechanics," Computer Methods in Applied Mechanics and Engineering, vol. 166, no. 1-2, pp. 3-24, 1998.

[27] A. Masud and R. A. Khurram, "A multiscale finite element method for the incompressible Navier-Stokes equations," Computer Methods in Applied Mechanics and Engineering, vol. 195, no. 13-16, pp. 1750-1777, 2006.

[28] S. K. Lele, "Compact finite difference schemes with spectral-like resolution," Journal of Computational Physics, vol. 103, no. 1, pp. 16-42, 1992.

[29] T. K. Sengupta and Y. G. Bhumkar, "New explicit two-dimensional higher order filters," Computers and Fluids, vol. 39, no. 10, pp. 1848-1863, 2010.

[30] A. Hashemian and H. M. Shodja, "A meshless approach for solution of Burgers' equation," Journal of Computational and Applied Mathematics, vol. 220, no. 1-2, pp. 226-239, 2008. 


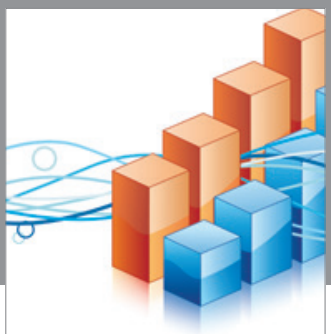

Advances in

Operations Research

vatem alat4

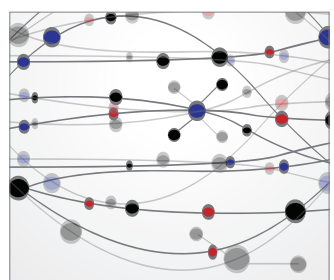

\section{The Scientific} World Journal
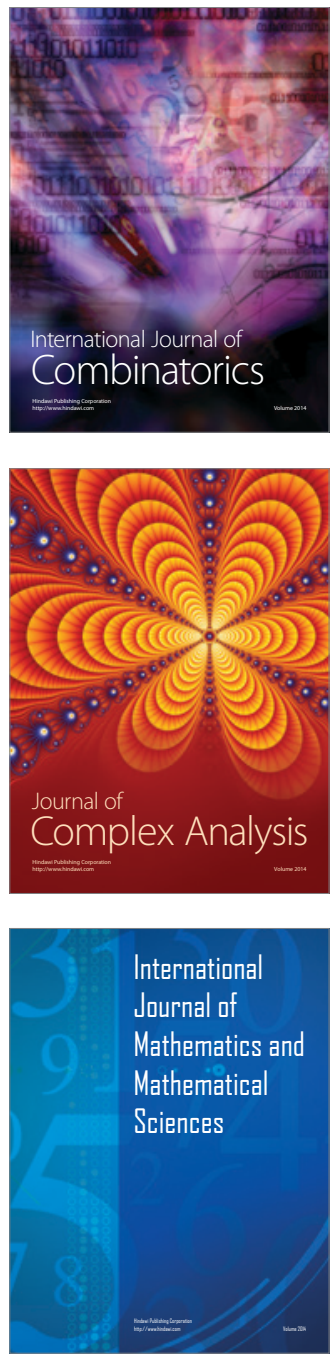
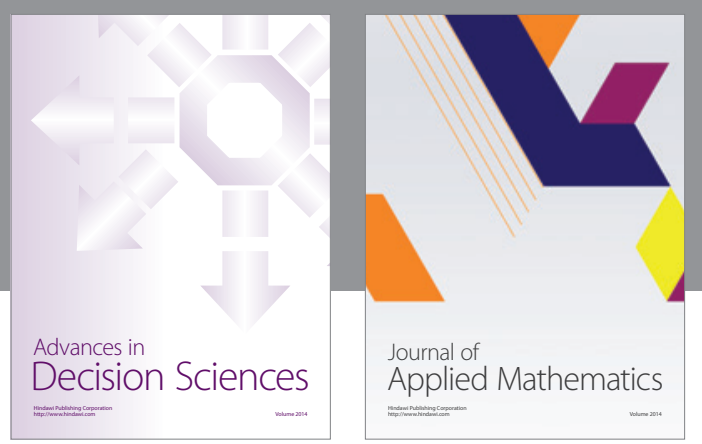

Algebra



\section{Hindawi}

Submit your manuscripts at

http://www.hindawi.com
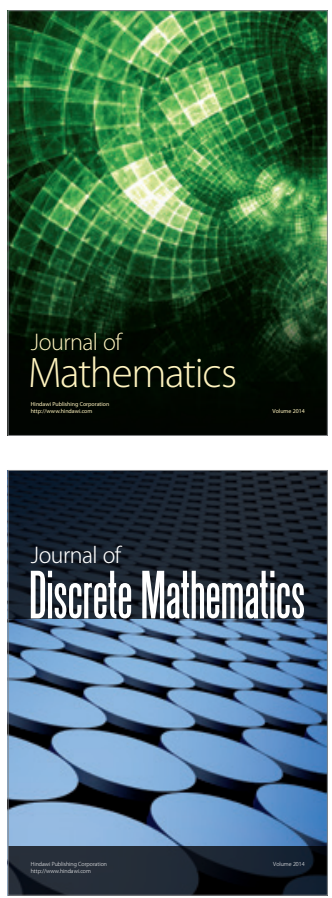

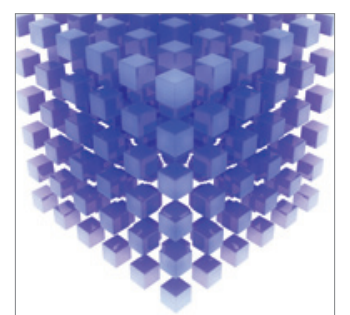

Mathematical Problems in Engineering
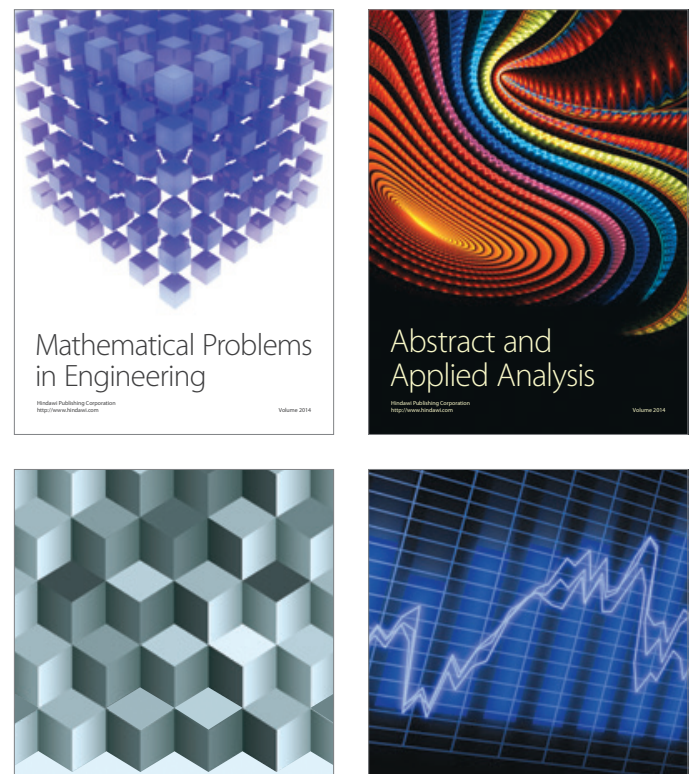

Journal of

Function Spaces



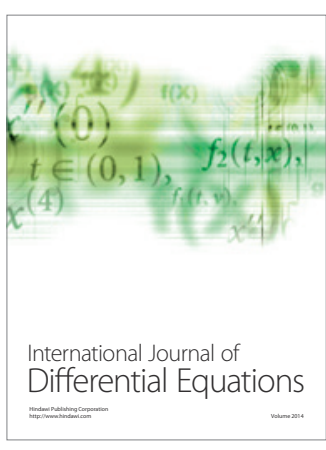
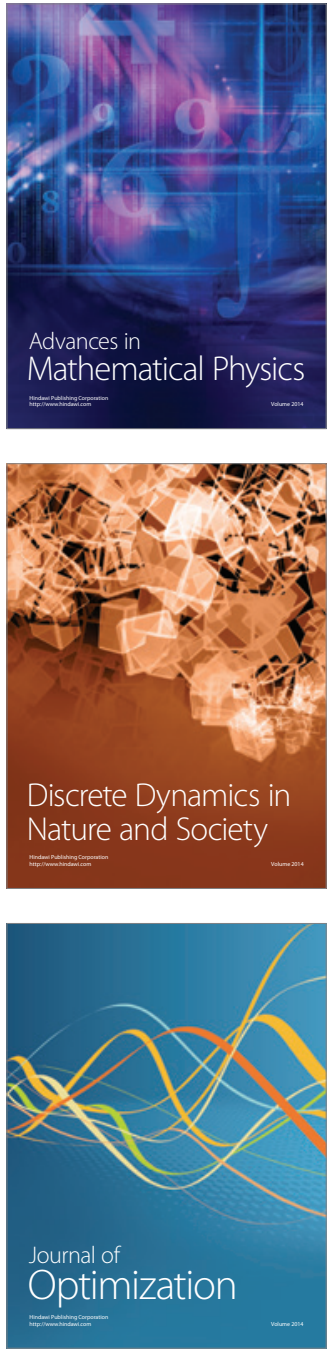